\title{
Epilepsy surgery in COVID times-a unique conundrum
}

\author{
Mohit Agrawal $^{1} \cdot$ Manjari Tripathi $^{2} \cdot$ Raghu Samala $^{1} \cdot$ Ramesh Doddamani $^{1} \cdot$ Bhargavi Ramanujan $^{2} \cdot$ P. Sarat Chandra ${ }^{1}$
}

Received: 28 December 2020 / Accepted: 14 January 2021 / Published online: 10 April 2021

(C) The Author(s), under exclusive licence to Springer-Verlag GmbH Germany, part of Springer Nature 2021

\begin{abstract}
The COVID-19 pandemic has forced hospitals to prioritize admissions. Epilepsy surgeries have been postponed at most centers. As the pandemic continues with no definite end in sight in the near future, the question arises until when such patients should be denied appropriate treatment. A 12-year-old child with left-sided Rasmussen's encephalitis with drug refractory epilepsy (DRE) presented at the height of the pandemic, with worsening of seizure frequency from 4-5/day to 20/day, with new-onset epilepsia partialis continua. She demonstrated features of progressive cognitive decline. The pros and cons of operating during the pandemic were discussed with the parents by a multidisciplinary team. She underwent endoscopic left hemispherotomy. Postoperatively she became seizure free but developed hospital-acquired mild COVID infection for which she was treated accordingly. Chosen cases of severe DRE, as the one illustrated above, who are deemed to benefit from surgery by a multidisciplinary team of physicians, should be re-categorized into the most severe class of patients and scheduled for surgery as soon as possible. The risk benefit ratio of the seizures being mitigated by surgery on one hand and possibility of acquiring COVID infection during hospital stay has to be balanced and a decision made accordingly.
\end{abstract}

Keywords Coronavirus $\cdot$ Drug refractory epilepsy $\cdot$ Endoscopic hemispherotomy $\cdot$ Infection

\section{Introduction}

Surgical admissions were prioritized during the COVID-19 pandemic $[9,12,15,17,22]$. Barring acute life-threatening conditions, most routine surgical cases were recommended to be postponed. One such group of patients were those suffering from drug refractory epilepsy (DRE) $[9,12,15,17,22]$. Epilepsy is a chronic condition, but a subset of patients in this group have multiple seizures daily [14]. Risks of continuing seizures include unexpected injuries, epileptic encephalopathy, status epilepticus, side effects of anti-epileptic drugs (AEDs), and the cumulative risk of sudden unexpected death in epilepsy patients $[8,10,14]$. This assumes more importance in a child suffering with daily seizures, especially when evaluation reveals a condition which may be amenable to surgical correction [21]. Surgery has not only been shown to be

P. Sarat Chandra

saratpchandra3@gmail.com

1 Department of Neurosurgery, Neurosciences Centre, All India Institute of Medical Sciences, Room No. 607, New Delhi 110029, India

2 Department of Neurology, All India Institute of Medical Sciences, New Delhi, India treatment of choice in pediatric patients suffering from DRE, but it has also shown to greatly improve cognitive outcomes $[4,11]$. Hence, it has been recommended for all patients with DRE to undergo early evaluation for possible epilepsy surgery [20]. As the COVID-19 pandemic continues, the question arises until when such patients should be denied appropriate treatment. We present a case which illustrates this dilemma which has been faced by epilepsy care givers in their day-to-day practice over the past few months.

\section{Case report}

- Clinical presentation: A 12-year girl had been having focal motor seizures involving the right half of the body with progressive hemiparesis since the past 4 years. On the basis of extensive evaluation, she had been diagnosed with Rasmussen's encephalitis with DRE and was planned to undergo left hemispherotomy, which was postponed because of the restrictions imposed due to COVID-19. During the pandemic, despite receiving five AEDs in appropriate doses, her seizure frequency worsened, and she developed epilepsia partialis continua (EPCs) involving the right hand. She became severely incapacitated and 
completely dependent on her parents to carry out her daily activities.

- Preoperative investigations: The child was admitted, and a video electroencephalogram (VEEG) and MRI were repeated (Figs. 1 and 2). Ancillary investigations had been done before the pandemic (Fig. 3). Neuropsychological assessment revealed a co-operative and communicative child with mild impairment of intellectual ability, mild memory impairment, and occasional behavioral abnormalities in the form of anger outbursts.

- Decision regarding surgical intervention: The case was discussed in a multi-disciplinary meeting. Considering the rapidly deteriorating general condition of the patient, it was decided to go ahead with immediate surgery. Her parents were counseled regarding the special protocols in place to mitigate the risk of iatrogenic COVID infection, namely, pre-admission COVID testing, restriction upon the number of hospital visitors, wearing masks and following hand hygiene protocols, a repeat preoperative COVID test, and the risk of postoperative infection.

- Surgery and postoperative course: The patient underwent endoscopic-assisted left hemispherotomy [6, 7], under all recommended precautions (Fig. 4a, b). She had an expected worsening of hemiparesis. She had no postoperative seizures. On day 12 after surgery, she developed diarrhea. A repeat COVID testing was conducted for which she

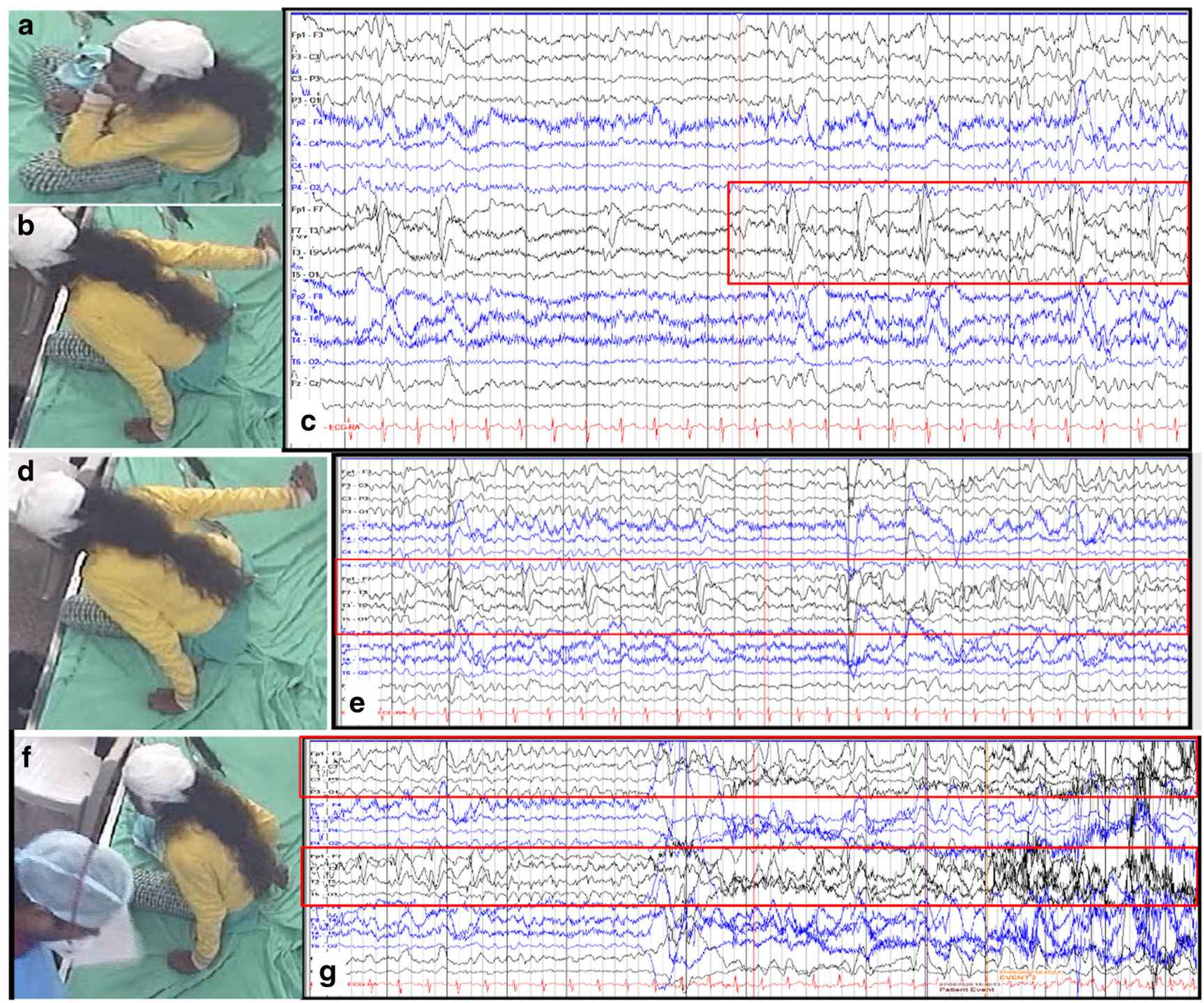

Fig. 1 (a) Child is sitting normally, while in (b), her right arm is seen going backward in a dystonic manner. The corresponding EEG (c) shows rhythmic spike and slow waves seen at a higher frequency, along with some lower amplitude rhythmic activity in the theta range. (d) The child has sustained posturing of the right arm in the ongoing seizure. (e) The corresponding EEG shows well-evolved rhythmic slow waves in the left frontal and temporal chains. (f) The seizure has ended here, obvious by the right arm of the child now by her side and recovered from the dystonic posturing. (g) In the first half of the panel, the left-sided channels continue to have rhythmic delta activity, which become higher in amplitude and even slower as the seizure ends, in the second half of the epoch shown 
Fig. 2 (a) Axial T1W contrastenhanced scan of the patient performed 2 years ago shows atrophy of the ipsilateral caudate head with consequent ventricular dilatation. (b) Coronal T1W contrastenhanced image shows left hippocampal atrophy and dilated temporal horn. There is absence of any contrast enhancement in either images. (c) Recent axial fluid-attenuated inversion recovery (FLAIR) image of the patient performed at the time of present admission shows progressive left hemispheric atrophy which has increased as compared to the previous scan. (d) Coronal FLAIR image demonstrates the perisylvian and hippocampal atrophy

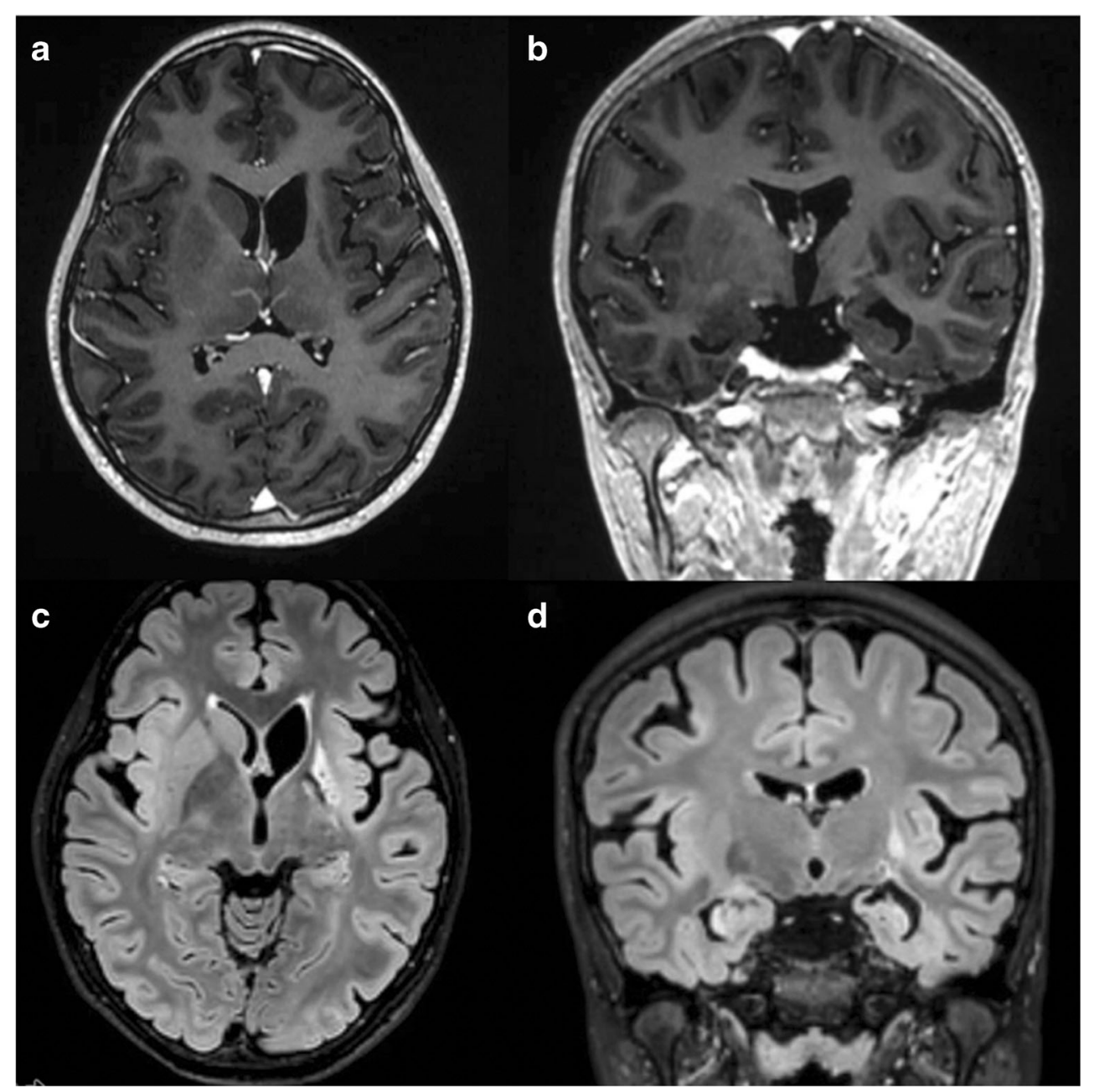

tested positive. As per protocol, she was immediately transferred to the designated COVID hospital of our institute. She maintained oxygen saturation more than $95 \%$ on room air and respiratory rate remained normal, she had no fever, and inflammatory markers and chest radiographs were also within the normal limits (Fig. 4c). She was categorized as having mild COVID infection and kept under observation. All her primary high-risk contacts tested negative, except for her physiotherapist who developed fever and tested positive for the virus 3 days later. At 3 months of follow-up, patient has remained seizure free and is able to walk with support.

\section{Discussion}

Epilepsy care services were severely affected across the board by the COVID-19 pandemic [1, 2, 13, 19]. However, the needs of the epilepsy patient community remained unchanged or even worsened by the imposition of lockdown and movement restrictions in several countries $[1,2,13,19]$. Therefore, there is an urgent need to identify the patients with DRE most at risk and prioritize their management. Among this subset of patients, those who can potentially benefit from undergoing epilepsy surgery should be identified and fast forwarded for appropriate management.

Our patient was at the brink of potentially irreversible deterioration in cognition, quality of life, as well as the risk posed due to high frequency of seizures. Rasmussen's encephalitis is a progressive disease, and surgery in the form of functional hemispherotomy is the only definitive treatment, following which seizure-free outcomes of 70-80\% have been consistently demonstrated [5]. Such a high-documented seizure freedom rate was one of the factors which gave us confidence to go ahead with surgery.

The risk benefit ratio of the seizures being mitigated by surgery on one hand and possibility of acquiring COVID infection during hospital stay has to be balanced. Fear of the risk of seizure worsening as a potential effect of COVID infection has remained largely unfounded. In a study from three tertiary care epilepsy hospitals from Spain and Italy, among the 5700 patients with epilepsy, only 14 tested positive for the virus. Further, there was no observed detrimental effect of the infection on the course of epilepsy [13]. Similarly, majority of the 


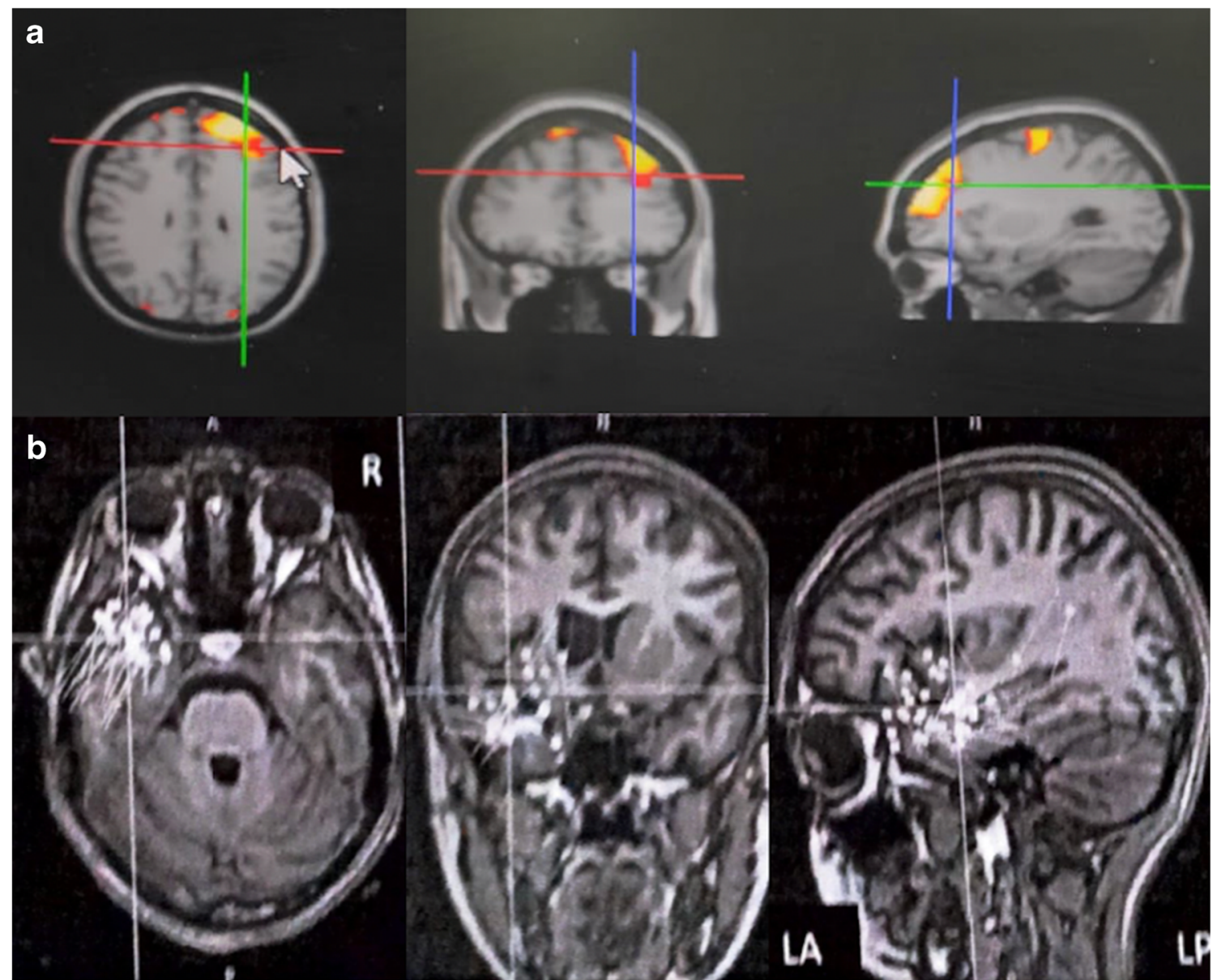

Fig. 3 (a) SISCOM localized the seizure focus to the left frontal and parietal region. (b) MEG showed dipoles clustered in left temporal lobe and mildly extending to left anterior/inferior/basi frontal region

337 respondents among members of the American Epilepsy Society noted to effect of COVID-19 infection in persons with epilepsy. A small percentage (17\%) noted worsening [2]. Rather than a direct effect of the viral infection, this worsening has been postulated to be due to the reduced seizure threshold associated with fever, increased stress levels, reduced sleep, and decreased drug compliance $[18,23]$. As the pandemic has progressed, a greater understanding of the disease pathophysiology and infection prevention strategies has now been obtained [16].

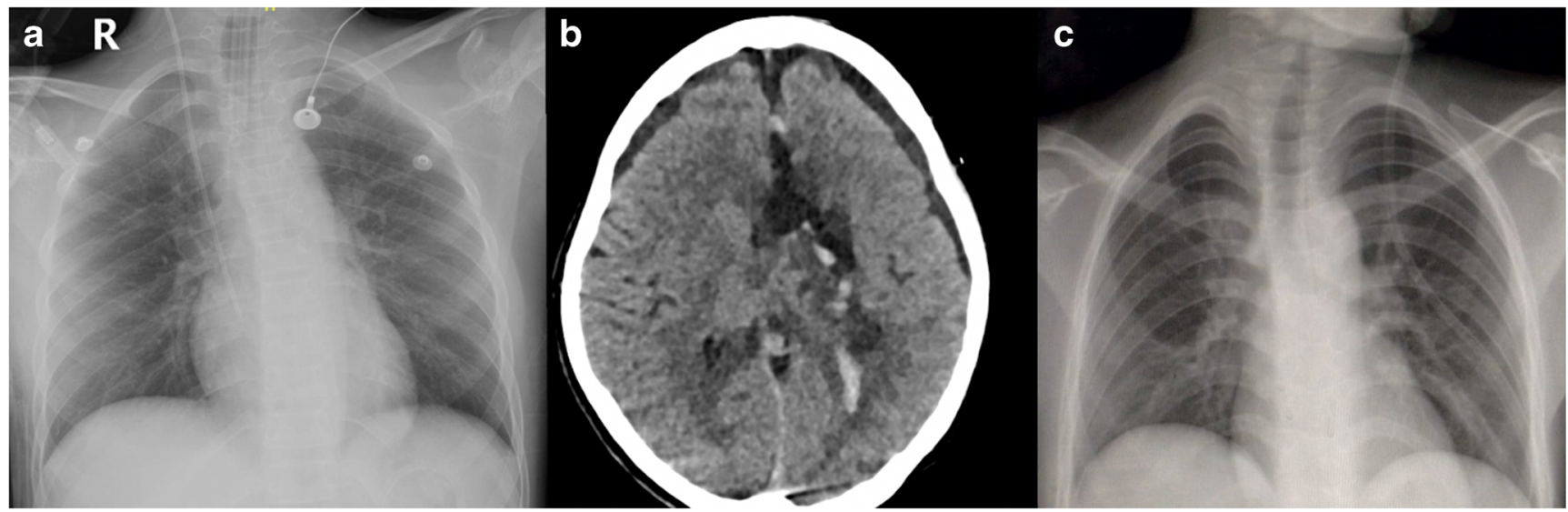

Fig. 4 (a) Immediate postoperative chest radiograph, (b) postoperative CT scan, and (c) chest radiograph after the diagnosis of COVID-19 was confirmed 


\section{Conclusion}

In an editorial published in the Journal of Neurosurgery at the start of this pandemic, it was suggested that every surgeon who confronts a possible surgical case in this time should ask herself/himself, "Is this patient likely to be able to safely wait for things to return to normal without incurring further neurological deficit and/or progression of disease to a point where it is less amenable to treatment?" If the answer to the above question was negative, it was recommended to advocate as strongly as possible to go ahead with surgery for such a patient [3]. Chosen cases of severe DRE, as the one illustrated above, should be re-categorized into the most severe class of patients and scheduled for surgery as soon as possible.

\section{Author contribution}

- Conceptualization: Mohit Agrawal, Manjari Tripathi, Ramesh Doddamani, and P. Sarat Chandra

- Methodology: Mohit Agrawal, Manjari Tripathi, Raghu Samala, Ramesh Doddamani, Bhargavi Ramanujan, Madhavi Tripathi, Chandrasekhar Bal, Ajay Garg, and P. Sarat Chandra

- Formal analysis and investigation: Mohit Agrawal, Manjari Tripathi, Raghu Samala, Ramesh Doddamani, Bhargavi Ramanujan, Madhavi Tripathi, Chandrasekhar Bal, Ajay Garg, and P. Sarat Chandra

- Writing - original draft preparation: Mohit Agrawal and P. Sarat Chandra

- Writing - review and editing: Ramesh Doddamani, Manjari Tripathi, and P. Sarat Chandra

- Supervision: Manjari Tripathi and P. Sarat Chandra

Data availability Not applicable.

\section{Compliance with ethical standards}

Conflict of interest On behalf of all authors, the corresponding author states that there is no conflict of interest.

Ethics approval Not applicable.

Informed consent The authors certify that they have obtained all appropriate patient consent forms. In the form, the patient has/have given his/ her/their consent for his/her/their images and other clinical information to be reported in the journal. The patients understand that their names and initials will not be published and due efforts will be made to conceal their identity.

Consent for publication Appropriate consent taken.

Code availability Not applicable.

\section{References}

1. Adan GH, Mitchell JW, Marson T (2020) Epilepsy care in the COVID-19 era. Clin Med (Lond) 20(4):e104-e1e6

2. Albert DVF, Das RR, Acharya JN, Lee JW, Pollard JR, Punia V, Keller JA, Husain AM (2020) The impact of COVID-19 on epilepsy care: a survey of the American Epilepsy Society Membership. Epilepsy Curr 20(5):316-324
3. Bernstein M (2020) Editorial. Neurosurgical priority setting during a pandemic: COVID-19. J Neurosurg:1-2

4. Braun KPJ (2020) Influence of epilepsy surgery on developmental outcomes in children. Eur J Paediatr Neurol 24:40-42

5. Cay-Martinez KC, Hickman RA, McKhann Ii GM, Provenzano FA, Sands TT (2020) Rasmussen encephalitis: an update. Semin Neurol 40(2):201-210

6. Chandra PS, Kurwale N, Garg A, Dwivedi R, Malviya SV, Tripathi M (2015) Endoscopy-assisted interhemispheric transcallosal hemispherotomy: preliminary description of a novel technique. Neurosurgery 76(4):485-494 discussion 94-95

7. Chandra PS, Subianto H, Bajaj J, Girishan S, Doddamani R, Ramanujam B, Chouhan MS, Garg A, Tripathi M, Bal CS, Sarkar C, Dwivedi R, Sapra S, Tripathi M (2018) Endoscopeassisted (with robotic guidance and using a hybrid technique) interhemispheric transcallosal hemispherotomy: a comparative study with open hemispherotomy to evaluate efficacy, complications, and outcome. J Neurosurg Pediatr 23(2):187-197

8. Dabla S, Puri I, Dash D, Vasantha PM, Tripathi M (2018) Predictors of seizure-related injuries in an epilepsy cohort from North India. J Epilepsy Res 8(1):27-32

9. Deora H, Sadashiva N, Tripathi M, Yagnick NS, Mohindra S, Batish A, Patil NR, Aggarwal A, Jangra K, Bhagat H, Panda N, Panigrahi M, Behari S, Chandra PS, Shukla DP, Singh L, Math SB, Gupta SK (2020) The aftermath of COVID-19 lockdown- why and how should we be ready? Neurol India 68:774-791

10. Devinsky O, Hesdorffer DC, Thurman DJ, Lhatoo S, Richerson G (2016) Sudden unexpected death in epilepsy: epidemiology, mechanisms, and prevention. Lancet Neurol 15(10):1075-1088

11. Dwivedi R, Ramanujam B, Chandra PS, Sapra S, Gulati S, Kalaivani M, Garg A, Bal CS, Tripathi M, Dwivedi SN, Sagar R, Sarkar C, Tripathi M (2017) Surgery for drug-resistant epilepsy in children. N Engl J Med 377(17):1639-1647

12. Germano A, Raffa G, Angileri FF, Cardali SM, Tomasello F (2020) Coronavirus disease 2019 (COVID-19) and neurosurgery: literature and neurosurgical societies recommendations update. World Neurosurg 139:e812-e8e7

13. Granata T, Bisulli F, Arzimanoglou A, Rocamora R (2020) Did the COVID-19 pandemic silence the needs of people with epilepsy? Epileptic Disord 22(4):439-442

14. Guerrini R (2006) Epilepsy in children. Lancet 367(9509):499-524

15. Gupta P, Muthukumar N, Rajshekhar V et al (2020) Neurosurgery and neurology practices during the novel COVID-19 pandemic: a consensus statement from India. Neurol India 68(2):246-254

16. Kooshkaki O, Derakhshani A, Conradie AM, Hemmat N, Barreto SG, Baghbanzadeh A, Singh PK, Safarpour H, Asadzadeh Z, Najafi S, Brunetti O, Racanelli V, Silvestris N, Baradaran B (2020) Coronavirus disease 2019: a brief review of the clinical manifestations and pathogenesis to the novel management approaches and treatments. Front Oncol 10:572329

17. Noureldine MHA, Pressman E, Krafft PR, Greenberg MS, Agazzi S, van Loveren H, Alikhani P (2020) Impact of the COVID-19 pandemic on neurosurgical practice at an academic tertiary referral center: a comparative study. World Neurosurg 139:e872-e8e6

18. Parihar J, Tripathi M, Dhamija RK (2020) Seizures and epilepsy in times of coronavirus disease 2019 pandemic. J Epilepsy Res 10(1): 3-7

19. Sanchez-Larsen A, Gonzalez-Villar E, Diaz-Maroto I et al (2020) Influence of the COVID-19 outbreak in people with epilepsy: analysis of a Spanish population (EPICOVID registry). Epilepsy Behav 112:107396

20. Sugano H, Arai H (2015) Epilepsy surgery for pediatric epilepsy: optimal timing of surgical intervention. Neurol Med Chir (Tokyo) 55(5):399-406

21. Tripathi M, Ray S, Chandra PS (2016) Presurgical evaluation for drug refractory epilepsy. Int J Surg 36(Pt B):405-410 
22. Venkataram T, Goyal N, Dash C et al (2020) Impact of the COVID19 pandemic on neurosurgical practice in India: results of an Anonymized National Survey. Neurol India 68(3):595-602

23. Vohora D, Jain S, Tripathi M, Potschka H (2020) COVID-19 and seizures: Is there a link? [published online ahead of print, $2020 \mathrm{Sep}$ 17]. Epilepsia. https://doi.org/10.1111/epi.16656
Publisher's note Springer Nature remains neutral with regard to jurisdictional claims in published maps and institutional affiliations. 